\title{
Gravitational radiation from precessing accretion disks in gamma-ray bursts
}

\author{
G. E. Romero ${ }^{1,2, \star}$, M. M. Reynoso ${ }^{3, \star}$, and H. R. Christiansen ${ }^{4}$ \\ ${ }^{1}$ Instituto Argentino de Radioastronomía (IAR), CCT La Plata (CONICET), C.C.5, (1894) Villa Elisa, Buenos Aires, Argentina \\ e-mail: romero@iar-conicet.gov.ar \\ 2 Facultad de Ciencias Astronómicas y Geofísicas, Universidad Nacional de La Plata, Paseo del Bosque s/n, 1900 La Plata, Argentina \\ 3 Instituto de Investigaciones Físicas de Mar del Plata (CONICET - UNMdP), Facultad de Ciencias Exactas y Naturales, Universidad \\ Nacional de Mar del Plata, Dean Funes 3350, (7600) Mar del Plata, Argentina \\ ${ }^{4}$ State University of Ceará, Physics Dept., Av. Paranjana 1700, 60740-000 Fortaleza - CE, Brazil
}

Received 28 April 2010 / Accepted 30 August 2010

\begin{abstract}
Context. We study the precession of accretion disks in the context of gamma-ray burst inner engines.

Aims. Our aim is to quantitatively estimate the characteristics of gravitational waves produced by the precession of the transient accretion disk in gamma-ray bursts.

Methods. We evaluate the possible periods of disk precession caused by the Lense-Thirring effect using an accretion disk model that allows for neutrino cooling. Assuming jet ejection perpendicular to the disk plane and a typical intrinsic time-dependence for the burst, we find gamma-ray light curves that have a temporal microstructure similar to that observed in some reported events. The parameters obtained for the precession are then used to evaluate the production of gravitational waves.

Results. We find that the precession of accretion disks of outer radius smaller than $10^{8} \mathrm{~cm}$ and accretion rates above $1 M_{\odot} \mathrm{s}^{-1}$ could be detected by Advanced LIGO if they occur at distances of less than $100 \mathrm{Mpc}$.

Conclusions. We conclude that the precession of a neutrino-cooled accretion disk in long gamma-ray bursts can be probed by gravitational wave astronomy. Precession of the disks in short gamma-ray events is undetectable with the current technology.
\end{abstract}

Key words. gamma-ray burst: general - accretion, accretion disks - gravitational waves

\section{Introduction}

In the central engines of gamma-ray bursts (GRBs), accretion onto a black hole resulting from the collapse of a massive star (e.g. Woosley 1993) or a merger of two compact objects (e.g. Mochkovitch et al. 1993) leads to the formation of a hot and dense, transient accretion disk. This disk can be significantly cooled by neutrino losses. The accretion of matter, at a rate $\sim 0.1-10 M_{\odot} \mathrm{s}^{-1}$, is supposed to power the burst, and the radiative processes in the relativistic jets are expected to account for the observed light curves. These curves display a wide variety of time profiles with timescales from milliseconds to minutes. The usual interpretation of this temporal structure is in terms of shocks that convert bulk kinetic energy into internal energy of the particles, which then cool by means of synchrotron and inverse Compton emission. The shocks can be internal to the jet and produced by colliding shells with different Lorentz factors (e.g. Kobayashi et al. 1997; Daigne \& Mochkovitch 1998; Guetta et al. 2001) or the result of interactions with the ambient medium (e.g. Heinz \& Begelman 1999; Fenimore et al. 1996). Among the observed light curves, however, there are some that are difficult to explain with the standard model (e.g. Romero et al. 1999). It has been suggested that the precession of the jet can play a role in the formation of the microstructure of both long and short gamma-ray bursts (e.g. Blackman et al. 1996; Portegies et al. 1999; Fargion 1999; Reynoso et al. 2006).

\footnotetext{
* Member of CONICET, Argentina.
}

Reynoso et al. (2006) developed a model for precessing jets based on spin-induced precession of the neutrino-cooled massive disk. The precession of the disk is transmitted to the relativistic jets, resulting in the peculiar temporal microstructure of some GRB's light curves.

In this paper, we study an additional effect of this precession of very massive accretion disks: the production of gravitational waves. Gravitational wave radiation is expected from gammaray bursts if the gravitational collapse is non-spherical, if there are strong inhomogeneities in the accretion disk, or, in the case of short GRBs, as the result of the spiraling and merging of compact objects (e.g. Mineshinge et al. 2002; Kobayashi \& Mészáros 2003).

The signal we consider here has a different origin from those previously discussed in the literature, and its specific features can shed light on the behavior of the innermost regions of the sources. We demonstrate that gravitational wave astronomy with instruments such as Advanced LIGO can be used to probe the Lense-Thirring effect in nearby GRBs.

\section{Accretion disks and spin-spin interaction in GRB engines}

Transient accretion disks are formed in GRB's engines such as collapsars and mergers of compact objects. The accretion rate in these disks is expected to vary significantly mostly in the outer part of the disk, while for the inner disk a constant accretion rate 
remains a valid approximation (e.g. Popham 1999; Di Matteo et al. 2005).

The conservation of mass falling with a velocity $v_{r} \simeq$ $r \sqrt{G M_{\mathrm{bh}} r^{-3}}$ at a radius $r$ from the black hole axis is given by

$\dot{M}=-2 \pi r v_{r} \Sigma(r)$,

where $\Sigma(r)=2 \rho(r) H(r)$ is the surface density, $H(r)$ is the disk half-thickness, and $\rho(r)$ is the mass density of the disk. The conservation of angular momentum and energy can be used to obtain numerically the functions $\Sigma(r)$ and $H(r)$, assuming that the heat generated by friction can be balanced by advection and neutrino emission (Reynoso et al. 2006). If the orbit of a particle around a spinning black hole is not aligned with the black hole equator, then the orbit precesses around the spinning axis. This is called the Lense-Thirring effect (Lense \& Thirring 1918), and originates from the dragging experienced by the inertial frames close to the rotating black hole. In accretion disks, the action of viscous torques leads to the alignment of the very inner part of the disk with the black hole equator plane (Bardeen \& Petterson 1975).

If the transient accretion disk in GRBs is formed misaligned with respect to the equator of a rapidly spinning black hole, then the accretion disk will develop precession. It has been argued that the disk precesses approximately like a rigid body, i.e., it does not present warping, when the disk Mach number is $\mathcal{M}<5$ (Nelson \& Papaloizou 2000). This condition is fulfilled in the accretion disks of GRBs. The precession of the disk leads to the precession of the jets, yielding a source of temporal variability and microstructure in the signal (Reynoso et al. 2006).

Neglecting any nutation movement, the precession period of the disk $\tau_{\mathrm{p}}$ can be related to its surface density as (see Liu \& Melia 2002; Caproni et al. 2004; Reynoso et al. 2006):

$\tau_{\mathrm{p}}=\int_{0}^{2 \pi} \frac{L_{\mathrm{d}}}{T_{\mathrm{d}}} \sin \theta \mathrm{d} \phi=2 \pi \sin \theta \frac{L_{\mathrm{d}}}{T_{\mathrm{d}}}$,

where the magnitudes of the disk angular momentum $L_{\mathrm{d}}$ and the precessional torque $T_{\mathrm{d}}$ applied to the disk are

$L_{\mathrm{d}}=2 \pi \int_{R_{\mathrm{ms}}}^{R_{\text {out }}} \Sigma(r) \Omega_{\mathrm{k}}(r) r^{3} \mathrm{~d} r$

$T_{\mathrm{d}}=4 \pi^{2} \sin \theta \int_{R_{\mathrm{ms}}}^{R_{\mathrm{out}}} \Sigma(r) \Omega_{\mathrm{k}}(r) v_{\mathrm{p}, \theta}(r) r^{3} \mathrm{~d} r$.

Here, if $a_{*}$ is the spin parameter,

$\Omega_{\mathrm{k}}(r)=\frac{c^{3}}{G M_{\mathrm{bh}}}\left[\left(\frac{r}{R_{\mathrm{g}}}\right)^{3 / 2}+a_{*}\right]^{-1}$

is the relativistic Keplerian angular velocity, $R_{\mathrm{g}}=G M_{\mathrm{bh}} / c^{2}$ is the gravitational radius, and

$v_{\mathrm{p}, \theta}=\frac{\Omega_{k}(R)}{2 \pi}\left[1-\sqrt{1 \mp 4 a_{*}\left(\frac{R_{\mathrm{g}}}{r}\right)^{1 / 2}+3 a_{*}^{2}\left(\frac{R_{\mathrm{g}}}{r}\right)^{2}}\right]$

is the nodal frequency obtained by perturbing a circular orbit in the Kerr metric (Kato 1990). The precessing part of the disk ends at an outer radius $R_{\text {out }}$, extending from an inner radius $R_{\mathrm{ms}}=$ $\xi_{\mathrm{ms}} R_{\mathrm{g}}$, where

$\xi_{\mathrm{ms}}=3+A_{2} \mp\left[\left(3-A_{1}\right)\left(3+A_{1}+2 A_{2}\right)\right]^{1 / 2}$,

with

$A_{1}=1+\left(1-a_{*}^{2}\right)^{1 / 3}\left[\left(1+a_{*}\right)^{1 / 3}+\left(1-a_{*}\right)^{1 / 3}\right]$,

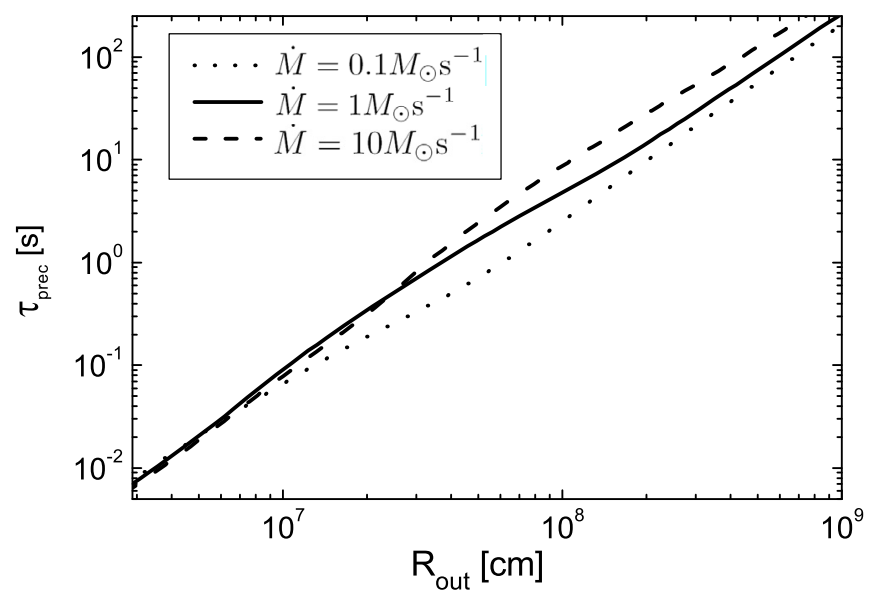

Fig. 1. Precession period as a function of the outer radius of the accretion disk for $\dot{M}=0.1 M_{\odot} \mathrm{s}^{-1}$ (dotted line), $\dot{M}=1 M_{\odot} \mathrm{s}^{-1}$ (solid line), and $\dot{M}=10 M_{\odot} \mathrm{s}^{-1}$ (dashed line).

and

$A_{2}=\left(3 a_{*}^{2}+A_{1}^{2}\right)^{1 / 2}$.

The minus sign in the expression for $\xi_{\mathrm{ms}}$ corresponds to prograde motion $\left(a_{*}>0\right)$, whereas the plus sign corresponds to retrograde motion $\left(a_{*}<0\right)$.

In Fig. 1, we show the precession period obtained as a function of the disk outer radius for different accretion rates.

\section{Gravitational waves from precessing disks in GRBs}

We attempted to characterize the emission of gravitational waves (GWs) from precessing accretion disks in GRB engines. Precession leads to observable features in the gamma-ray light curves. Its effects are illustrated in the following subsection, and the necessary formulae for GW emission are presented in Sect. 3.2.

\subsection{Specific models of precessing accretion disk in GRBs}

We assumed that the precession of accretion disks in GRBs is transmitted to the jets. The electromagnetic emission is generated in the jet by synchrotron and inverse Compton processes. The dependence of the gamma-ray luminosity on the angle with respect to the jet axis $\psi$ is described by the expression (Portegies-Swart et al. 1999)

$$
\begin{aligned}
L(\psi)= & \frac{27}{4}\left[\mathrm{e}^{-0.6 \Psi(x)}-\frac{8}{9}\right] \\
& \times\left[\mathrm{e}^{-0.3 \Psi(x)}-\mathrm{e}^{-6.3 \Psi(x)}\right] \frac{\mathrm{d} \Psi(x)}{\mathrm{d} x},
\end{aligned}
$$

where $x=10 \sin \psi$ and $\Psi(x)=\frac{1}{6} \ln \left(1+4 x^{2}\right)$. The intrinsic temporal dependence of the signal is characterized by a FRED (Fast Rise and Exponential Decay) behavior,

$I(t)=N_{I}\left(1-\mathrm{e}^{-\frac{t}{\tau_{\text {rise }}}}\right)\left\{\frac{\pi}{2}-\tan ^{-1}\left[\frac{t-\tau_{\text {plat }}}{\tau_{\text {dec }}}\right]\right\}$,

where $N_{I}$ is a normalization constant such that the maximum of the signal corresponds to unity, and $\tau_{\text {rise }}, \tau_{\text {plat }}$, and $\tau_{\text {dec }}$ are the timescales for the initial rise, plateau, and decay, respectively. 

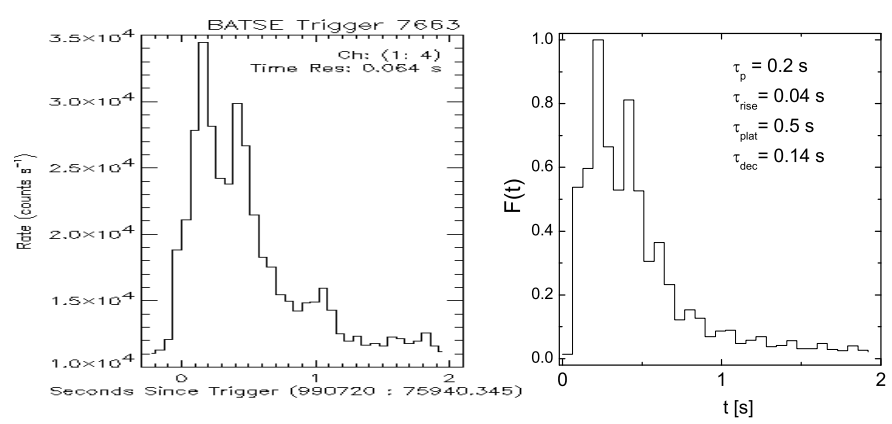

Fig. 2. GRB 990720 light curve (left panel) and light curve obtained with a precessing disk (right panel).
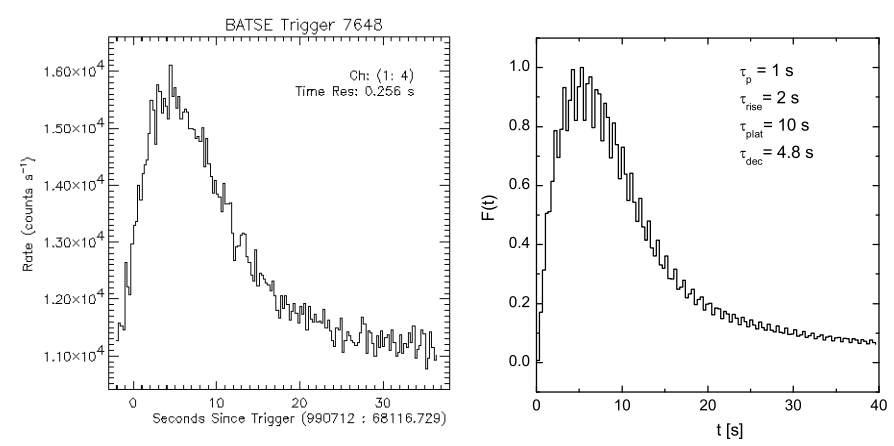

Fig. 3. GRB 990712 light curve (left panel) and light curve obtained with a precessing disk (right panel).

We considered two specific GRBs as examples: the short burst GRB 990720 and the long burst GRB 990712. For these bursts, we found a proper set of the relevant timescales and precession period. Then, using the model of Reynoso et al. (2006) we reproduced the observed light curves.

The original time profiles corresponding to the mentioned events and the light curves obtained using $F(t)=I(t) L(\psi(t))$ where the effect of precession is given by $\phi(t)=2 \pi\left(t / \tau_{\text {prec }}\right)$, are shown in Figs. 2 and 3. In both cases, the observer is located at $\theta_{\text {obs }}=2^{\circ}$ with respect to the $z$-axis perpendicular to the black hole equator, and the angle $\psi(t)$ between the jet and the observer is time-dependent because of the precession.

\subsection{General formulae for gravitational wave emission}

An axissymmetric body (i.e., with inertial moments $I_{1}=I_{2}$ ) in precession emits gravitational waves with an amplitude given by (Zimmermann \& Szedenits 1979; Maggiore 2008)

$h_{\text {prec }}(t)=h_{+}(t)+h_{\times}(t)$,

where

$h_{+}(t)=F_{+, 1} \cos \Omega t+F_{+, 2} \cos 2 \Omega t$,

$h_{\times}(t)=F_{\times, 1} \sin \Omega t+F_{\times, 2} \sin 2 \Omega t$,

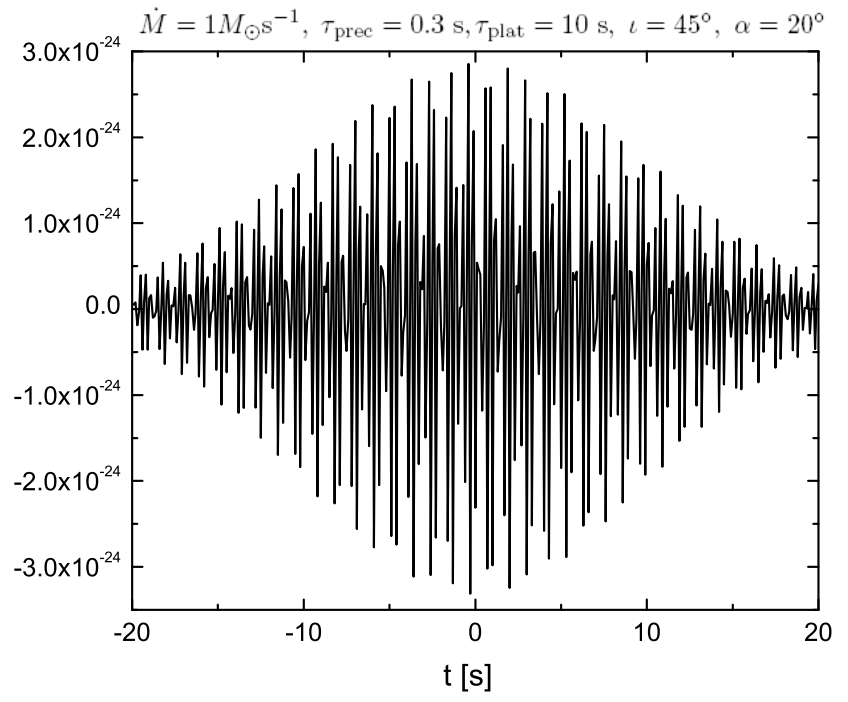

Fig. 4. A typical GW waveform that can be produced by a precessing GRB accretion disk. The accretion rate is $\dot{M}=1 M_{\odot} \mathrm{s}^{-1}$, the precession period is $\tau_{\text {prec }}=0.3 \mathrm{~s}$, and the duration of the event is given by the timescale $\tau_{\text {plat }}=10 \mathrm{~s}$.

with

$F_{+, 1}=h_{0}^{\prime} \sin 2 \alpha \sin \iota \cos \iota$

$F_{+, 2}=2 h_{0}^{\prime} \sin ^{2} \alpha\left(1+\cos ^{2} \iota\right)$

$F_{\times, 1}=h_{0}^{\prime} \sin 2 \alpha \sin \iota$

$F_{\times, 2}=4 h_{0}^{\prime} \sin ^{2} \alpha \cos \iota$,

and

$h_{0}^{\prime}=-\frac{G}{c^{4}} \frac{\left(I_{3}-I_{1}\right) \Omega^{2}}{d}$.

Here, $\alpha$ is the angle between the angular momentum of the disk and that of the black hole, $\iota$ is the angle between the $z$-axis of the detector and the signal direction of arrival, and $d$ is the distance to the radiating body. The principal inertia moments are

$I_{3}=\int_{V}\left(x^{2}+y^{2}\right) \rho(r)$

$I_{1}=\int_{V}\left(z^{2}+y^{2}\right) \rho(r)$.

The frequency of the gravitational waves are $f_{1}=\Omega /(2 \pi)$ and $f_{2}=2 \Omega /(2 \pi)$, which are related to the angular momentum of the body by

$\Omega=\frac{L_{\mathrm{d}}}{I_{1}}$.

Since we are dealing with bursting events, the GW signal is expected to be significant for a time $\tau_{\text {plat }}$. Therefore, we modulated the signal of Eq. (12) using a Gaussian

$h(t)=h_{\text {prec }}(t) \mathrm{e}^{-\frac{t^{2}}{2 \tau_{\text {plat }}^{2}}}$.

This is usually adopted to describe the GW signal from bursting sources (e.g. Acernese 2008; Maggiore 2004). The angular 

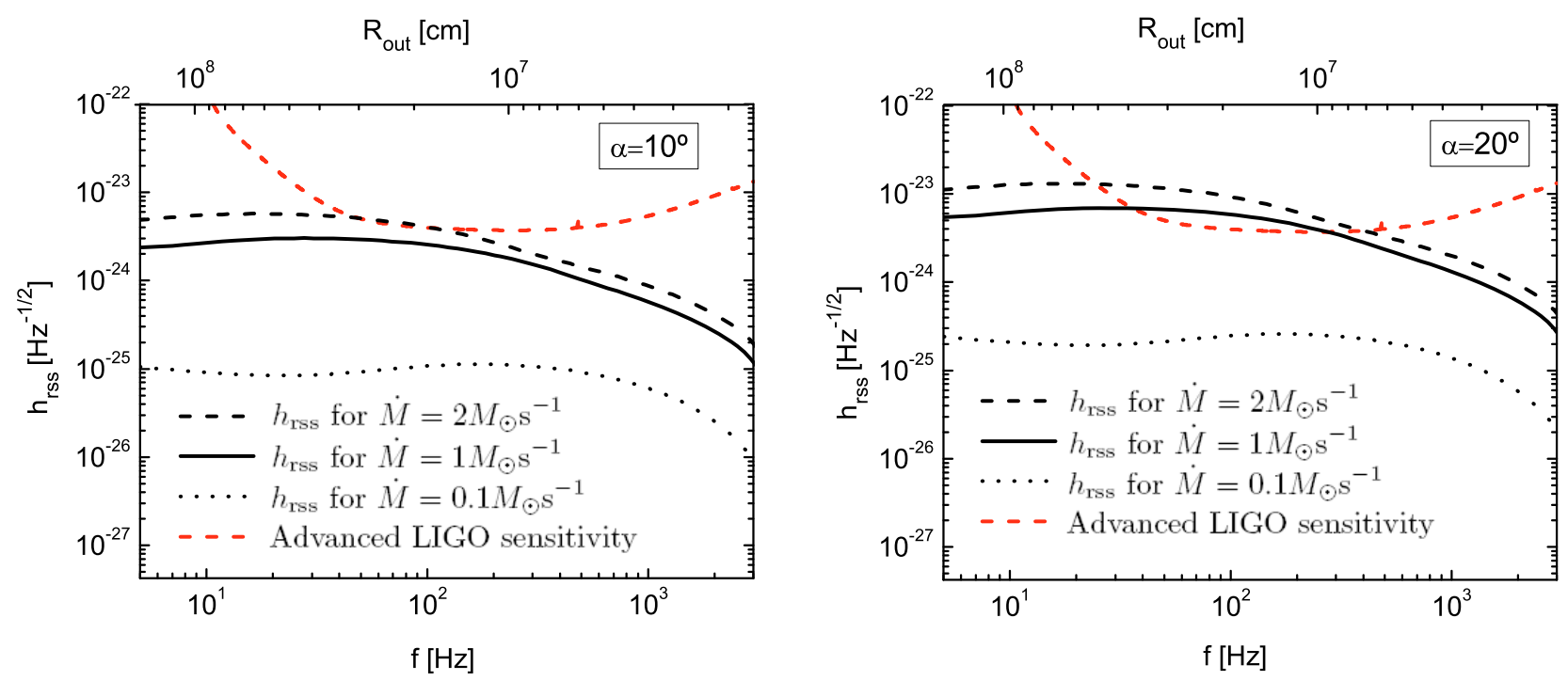

Fig. 5. Gravitational wave rss amplitude for different accretion rates and Advanced LIGO sensitivity (red dashed line) as a function of the gravitational wave frequency for $\alpha=10^{\circ}$ (left panel) and for $\alpha=20^{\circ}$ (right panel). The corresponding outer radius of the accretion disk is indicated in the upper horizontal axis.

frequencies that contribute to the waveform of Eq. (23) can be obtained from its Fourier transform $\tilde{h}(\omega)=\tilde{h}_{+}(\omega)+\tilde{h}_{\times}(\omega)$, where

$$
\begin{aligned}
\tilde{h}_{+}(\omega)= & \frac{\tau_{\text {plat }}}{2}\left[F_{+, 1}\left(\mathrm{e}^{\frac{-\tau_{\text {plat }}^{2}}{2}(\omega+\Omega)^{2}}+\mathrm{e}^{\frac{-\tau_{\text {plat }}^{2}}{2}(\omega-\Omega)^{2}}\right)\right. \\
& \left.+F_{+, 2}\left(\mathrm{e}^{\frac{-\tau_{\text {plat }}^{2}}{2}(\omega+2 \Omega)^{2}}+\mathrm{e}^{\frac{-\tau_{\text {plat }}^{2}}{2}(\omega-2 \Omega)^{2}}\right)\right]
\end{aligned}
$$

and

$$
\begin{aligned}
\tilde{h}_{\times}(\omega)= & \frac{i \tau_{\text {plat }}}{2}\left[F_{\times, 1}\left(\mathrm{e}^{\frac{-\tau_{\text {plat }}^{2}}{2}(\omega+\Omega)^{2}}+\mathrm{e}^{\frac{-\tau_{\text {plat }}^{2}}{2}(\omega-\Omega)^{2}}\right)\right. \\
& \left.+F_{\times, 2}\left(\mathrm{e}^{\frac{-\tau_{\text {plat }}^{2}}{2}(\omega+2 \Omega)^{2}}+\mathrm{e}^{\frac{-\tau_{\text {plat }}^{2}}{2}(\omega-2 \Omega)^{2}}\right)\right] .
\end{aligned}
$$

It can be seen from these expressions that the ranges of frequencies that contribute to the signal are centered around $\Omega$ and $2 \Omega$, and the width of the ranges is $\tau_{\text {plat }}^{-1}$. Given the typical durations of GRBs, the frequency spread is narrow for all bursts except for those with durations much shorter than $1 \mathrm{~s}$. In Fig. 4 we show an example waveform obtained with Eq. (23) for an accretion rate $\dot{M}=1 M_{\odot} \mathrm{s}^{-1}$, a precession period $\tau_{\text {prec }}=0.3 \mathrm{~s}, \tau_{\text {plat }}=10 \mathrm{~s}$, $\iota=45^{\circ}, \alpha=20^{\circ}$, and $d=100 \mathrm{Mpc}$.

\section{Detectability}

We now consider the gravitational waves produced by the disk precession in events with the characteristics of those discussed in the previous section. In Table 1, we list the main parameters for the short and long GRBs taken as examples. We estimated the amplitude of the gravitational waves using Eq. (19), considering different accretion rates. To assess the detectability, we calculated the root-sum-square amplitude (e.g. Acernese 2008; Maggiore 2004)

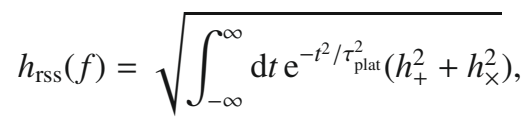

Table 1. Parameters used for the example GRB events.

\begin{tabular}{ccc}
\hline \hline Parameter & GRB 990712 & GRB 990720 \\
\hline$\tau_{\text {prec }}[\mathrm{s}]$ & 1.0 & 0.2 \\
$\tau_{\text {rise }}[\mathrm{s}]$ & 2.0 & 0.04 \\
$\tau_{\text {plat }}[\mathrm{s}]$ & 10.0 & 0.5 \\
$\tau_{\text {dec }}[\mathrm{s}]$ & 4.8 & 0.14 \\
$\dot{M}\left[M_{\odot} \mathrm{s}^{-1}\right]$ & $1-10$ & 0.1 \\
$R_{\text {out }}\left[10^{7} \mathrm{~cm}\right]$ & $3.3-3.7$ & 2.1 \\
\hline
\end{tabular}

where $f=\Omega /(2 \pi)$. For illustration, we choose the value $\iota=45^{\circ}$ for the angle between the line of sight and the $z$-axis of the detector, and we take $\alpha=10^{\circ}-20^{\circ}$ to be the angle between the angular momentum of the disk and that of the black hole. In Fig. 5 we plot the root-sum-square amplitude $h_{\text {rss }}$, as a function of one of the allowed frequencies, $f=\Omega /(2 \pi)$, and also as a function of the outer radius of the inner precessing disk. We use $\tau_{\text {plat }}=2 \mathrm{~s}$ for $\dot{M}=0.1 M_{\odot} \mathrm{s}^{-1}$, and $\tau_{\text {plat }}=10 \mathrm{~s}$ for $\dot{M}=1-2 M_{\odot} \mathrm{s}^{-1}$. In the figure, we include the expected sensitivity for Advanced LIGO (Shoemaker 2010).

The parameters used to find $\Sigma(r)$ and $H(r)$ are $M_{\mathrm{bh}}=3 M_{\odot}$, $a_{*}=0.1$, a viscosity parameter $\alpha=0.1$, and the different massloss rates $\dot{M}=\left\{0.1 M_{\odot} \mathrm{s}^{-1}, 1 M_{\odot} \mathrm{s}^{-1}, 2 M_{\odot} \mathrm{s}^{-1}\right\}$. The distance to the GRB is taken to be $d=100 \mathrm{Mpc}$.

As can be seen from Fig. 5, there are higher probabilities of detection for accretion rates higher than $1 M_{\odot} \mathrm{s}^{-1}$ and outer radii between $10^{7}$ and $10^{8} \mathrm{~cm}$. When the accretion rate is very high, the disk may become advection dominated rather than cooled by neutrino emission (Liu et al. 2008). The dynamics in the gravitational field, however, is not affected. High accretion rates can be sustained only in long GRBs, so we conclude that there is only a good prospect for detection of gravitational waves from precessing disks of nearby $(d<100 \mathrm{Mpc})$ and long events. These events are likely to be related to the death of very massive stars, so the host galaxies should have active star-forming regions. 
Two low-luminosity long GRBs (980425 and 060218) were already observed at distances of $\sim 40 \mathrm{Mpc}$ and $\sim 130 \mathrm{Mpc}$, respectively (Corsi \& Mészáros 2009). The local rate of long GRBs is estimated to be $\sim 200 \mathrm{Gpc}^{-3} \mathrm{yr}^{-1}$ (e.g. Liang et al. 2007; Virgili et al. 2009). INTEGRAL has detected a large proportion of faint GRBs that have been inferred to be local (Foley et al. 2008). All this suggests that the detection of precessing disks of GRBs through their gravitational emission is possible in the near future.

\section{Summary and conclusions}

We have studied long and short gamma-ray bursts showing that the presence of microvariability in their light curves can be caused by the precession of the transient accretion disk. We then considered precessing disks of GRBs as a source of GWs and estimated the resulting waveform produced by the phenomenon.

Our results indicate that if the outer radius of the precessing disk is between $10^{7}$ and $10^{8} \mathrm{~cm}$ and the accretion rate higher than $1 M_{\odot} \mathrm{s}^{-1}$, the gravitational waves can be detected from distances of $100 \mathrm{Mpc}$ or less. We conclude that only in relatively nearby and long GRBs can the precession of the accretion disk be studied by gravitational wave astronomy. The detection of one event of this class can be used to test the Lense-Thirring effect in the strong field limit.

Acknowledgements. We thank O. A. Sampayo for useful comments on GW physics. This research was supported by the Argentine Agencies CONICET and ANPCyT through grants PIP 112-200901-00078, PIP 112-200801-00587, and PICT-2007-00848 BID 1728/OC-AR. G.E.R. acknowledges additional support from the Ministerio de Educación y Ciencia (Spain) under grant AYA 2007-68034-C03-01, FEDER funds, and HRC acknowledges financial support of FUNCAP, Brazil.

\section{References}

Acernese, F., et al. 2008, Class. Quant. Grav., 25, 225001

Bardeen, J. M., \& Petterson, J. A. 1975, ApJ, 195, L65

Blackman, E. G., Yi, I., \& Field, G. B. 1996, ApJ, 473, L79

Caproni, A., Mosquera Cuesta, H. J., \& Abraham, Z. 2004, ApJ, 616, L99

Corsi, A., \& Mészáros, P. 2009, ApJ, 702, 1171

Daigne, F., \& Mochkovitch, R. 1998, MNRAS, 296, 275

Di Matteo, T., Perna, R., \& Narayan, R. 2002, ApJ, 579, 706

Fargion, D. 1999, in 26th ICRC, OG2.3.14, ed. D. Kieda, M. Salamon, \& B. Dingus, 32

Foley, S., McGlynn, S., Hanlon, L., McBreen, S., \& McBreen, B. 2008, A\&A, 484, 143

Guetta, D., Spada, M., \& Waxman, E. 2001, ApJ, 557, 399

Heinz, S., \& Begelman, M. C. 1999, ApJ, 527, L35

Kato, S. 1990, PASJ 42, 99

Kobayashi, S., \& Mészáros, P. 2003, ApJ, 589, 861

Kobayashi, S., Piran, T., \& Sari, R. 1997, ApJ, 490, 92

Lense, J., \& Thirring, H. 1918, Phys. Z., 19, 156

Liang, E., Zhang, B., Virgili, F., \& Dai, Z. G. 2007, ApJ, 662, 1111

Liu, S., \& Melia, F. 2002, ApJ, 573, L23

Liu, T., Gu, W.-M., Xue, L., Weng, S.-S., \& Lu, J.-F. 2008, ApJ, 676, 545

Maggiore, M. 2008, Gravitational waves, Theory and Experiments (New York: Oxford University Press), Vol. 1

Mineshinge, S., Hosohawa, T., Machida, M., \& Matsumoto, R. 2002, PASJ, 54, 655

Mochkovitch, R., Hernanz, M., Isern, J., \& Martin, X. 1993, Nature, 361, 236

Nelson, R. P., \& Papaloizou, J. C. B. 2000, MNRAS, 315, 570

Popham, R., Woosley, S. E., \& Fryer, C. 1999, ApJ, 518, 356

Portegies-Zwart, S. F., Lee, C. H., \& Lee, H. K. 1999, ApJ, 520, 666

Romero, G. E., Torres, D. F., Andruchow, I. \& Anchordoqui, L. A. 1999 MNRAS, 308, 799

Reynoso, M. M., Romero, G. E., \& Sampayo, O. A. 2006, A\&A, 454, 11

Shoemaker, D. (LIGO Scientific Collaboration) 2010, LIGO document T0900288-v3, http://dcc.ligo.org/cgi-bin/DocDB/ShowDocument? docid $=2974$

Virgili, F. J., Liang, E.-W., \& Zhang, B. 2009, MNRAS, 392, 91

Woosley, S. E. 1993, ApJ, 405, 273

Zimmermann, M., \& Szedenits, E. 1979, Phys. Rev. D, 20, 351 